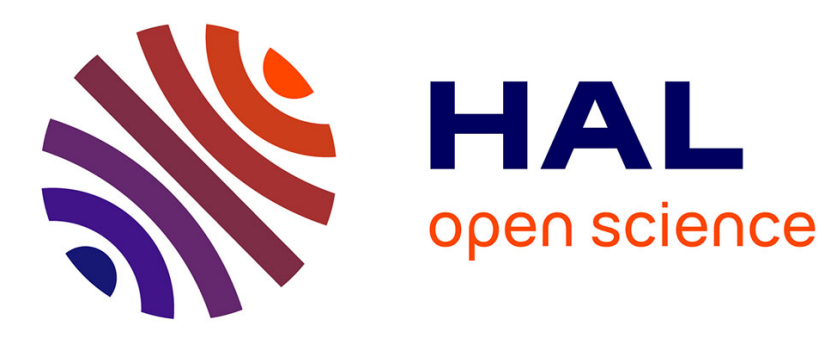

\title{
Bifurcation Analysis and Chaos in Simplest Fractional-order Electrical Circuit
}

\author{
Mohammed-Salah Abdelouahab, René Lozi
}

\section{To cite this version:}

Mohammed-Salah Abdelouahab, René Lozi. Bifurcation Analysis and Chaos in Simplest Fractionalorder Electrical Circuit. IEEE 3rd International Conference on Control, Engineering \& Information Technology (CEIT), May 2015, Tlemcen, Algeria. pp.1-5, 10.1109/CEIT.2015.7233122 . hal01337180

\section{HAL Id: hal-01337180 \\ https://hal.science/hal-01337180}

Submitted on 24 Jun 2016

HAL is a multi-disciplinary open access archive for the deposit and dissemination of scientific research documents, whether they are published or not. The documents may come from teaching and research institutions in France or abroad, or from public or private research centers.
L'archive ouverte pluridisciplinaire HAL, est destinée au dépôt et à la diffusion de documents scientifiques de niveau recherche, publiés ou non, émanant des établissements d'enseignement et de recherche français ou étrangers, des laboratoires publics ou privés. 


\section{Bifurcation Analysis and Chaos in Simplest Fractional-order Electrical Circuit}

\author{
Mohammed-Salah Abdelouahab \\ Laboratory of Mathematics and their interactions \\ Mila University Center, Mila, Algeria \\ Email: m.abdelouahab@centre-univ-mila.dz
}

\begin{abstract}
In this paper we investigate the bifurcation and chaos in a fractional-order simplest electrical circuit composed of only three circuit element: a linear passive capacitor, a linear passive inductor and a nonlinear active memristor with two degree polynomial memristance and a second order exponent internal state. It is shown that this fractional circuit can exhibit rich nonlinear dynamics such as a Hopf bifurcation, double scroll chaotic attractor, four scroll chaotic attractor and new chaotic attractor which is not observed in the integer case. Finally, the presence of chaos is confirmed by the application of the recently introduced 0-1 test.
\end{abstract}

\section{INTRODUCTION}

The fractional calculus is more than 300 years old with the first written note dated to 1695 [1]. Several physical phenomena can be described more accurately by fractional differential equations rather than integer-order models [2]. In the past, the lack of methods for solving fractional differential equations was the reason for using only integer-order models. Nowadays, a number of techniques are available for approximating fractional derivatives and integrals [3].

There are several definitions of fractional derivatives and integrals [4], for example for a sufficiently smooth function $f:$

The Riemann-Liouville fractional integral of order $\alpha>0$ is given by

$$
J_{a}^{\alpha} f(t)=\frac{1}{\Gamma(\alpha)} \int_{a}^{t}(t-s)^{\alpha-1} f(s) d s, \quad t>a .
$$

The Riemann-Liouville fractional-order derivative ${ }_{a}^{R L} D_{t}^{\alpha} f$ is defined by

$$
{ }_{a}^{R L} D_{t}^{\alpha} f=D^{m} J_{a}^{m-\alpha} f, m=\lceil\alpha\rceil
$$

The Caputo fractional-order derivative $a D_{t}^{\alpha} t$ is defined by

$$
{ }_{a} D_{t}^{\alpha} f(t)=J_{a}^{m-\alpha} D^{m} f(t), m=\lceil\alpha\rceil
$$

The Grünwald-Letnikov fractional-order derivative is given by

$$
{ }_{a} L D_{t}^{\alpha} f(t)=\lim _{h \rightarrow 0} h^{-\alpha} \sum_{k=0}^{\frac{t-a}{h}}(-1)^{k}\left(\frac{\Gamma(\alpha+1)}{k ! \Gamma(\alpha-k+1)}\right) f(t-k h) .
$$

Fractional-order derivatives of a periodic function cannot be a periodic function [5], as a consequence of this property, the time-invariant fractional-order systems do not have any non-constant periodic solution. In [6] we have

\author{
René Lozi \\ Laboratory of Mathematics J. A. Dieudonné \\ U.M.R. CNRS 7351, University of Nice-Sophia Antipolis, \\ Parc Valrose, 06108 Nice Cedex 02, France \\ r.lozi@unice.fr
}

proposed a solution for this problem by imposing a simple modification to the Grünwald-Letnikov definition.

Memristor is a new electrical element which has been predicted and described in 1971 by Leon O. Chua [8] and for the first time realized by HP laboratory in 2008 [9]. Chua proved that memristor behavior could not be duplicated by any circuit built using only the other three elements (resistor, capacitor, and inductor) In [7] we have generalized the definition of fractance (which was first introduced in 1983) and after that, introduced the paradigm of memfractance which is fitted for circuit elements with memory such as memristor, meminductor, memcapacitor and second-order memristor. We have defined a new element called memfractor which possesses interpolated characteristics between those four circuit elements and proved a generalized Ohm's law. Due to the nonlinearity of memristor element, memristor-based circuits can easily generate a chaotic signal. In 2010, Muthuswamy and Chua [10] proposed a memristor based circuit comprising only three elements: a linear passive inductor, a linear passive capacitor, and a nonlinear active memristor with a second degree polynomial memristance

$$
M(z(t))=\beta\left(z^{2}(t)-1\right)
$$

connected in series, which has been shown to be the simplest circuit capable of generating a one scroll chaotic attractor. In order to generate a double scroll and a four scroll chaotic attractor the authors Teng et al. [11] replaced the second degree polynomial memristance by a fourth degree polynomial memristance

$$
M(z(t))=\delta z^{4}(t)+\gamma z^{2}(t)-\beta
$$

and set the exponent of the internal state function of memristor to second order.

$$
\dot{z}=-i_{L}(t)-\alpha z(t)+i_{L}^{2}(t) z(t) .
$$

In this paper we investigate the bifurcation and chaos in a fractional-order version of the proposed memristorbased simplest chaotic circuit with two degree polynomial memristance and a second order exponent internal state.

\section{Simplest MEMRISTOR-BASED CHAOTIC CIRCUIT}

The proposed simplest circuit in this paper can generate a double scroll Fig.(1-a) and a four-scroll chaotic attractor Fig.(1-b) by using only a second degree polynomial memristance as in [10] and setting the exponent 


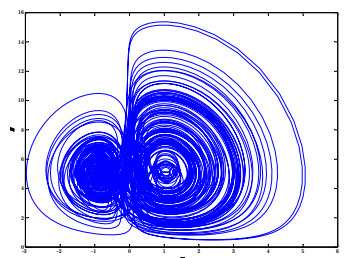

(a)

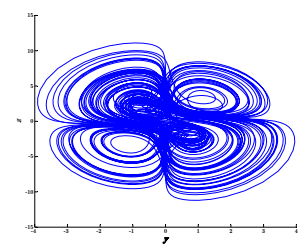

(b)
Fig. 1. a) Double scroll chaotic attractor for $L=3 H, C=1 F, \alpha=$ $0.9 \beta=10.1, \gamma=0.4$ b) 4 -scroll chaotic attractor for $L=3 H, C=$ $1 F \alpha=0.9, \beta=3, \gamma=0.4$.

of the internal state function of the memristor to second order. The dynamic of the circuit is described by the mathematical model

$$
\left\{\begin{array}{l}
\dot{x}=a y \\
\dot{y}=-b(x+M(z)) y \\
\dot{z}=-y-\alpha z+y^{2} z
\end{array}\right.
$$

where $x(t)=V(t)$ is the voltage across the capacitor, $y(t)=I_{L}(t)$ is the current through the inductor, $z(t)$ denotes the internal state variable of the memristor, $a=$ $1 / C$ the is inverse capacitance, $b=1 / L$ is the inverse inductance and the memristor function is given by

$$
M(z(t))=\gamma z^{2}(t)-\beta
$$

The simulation of system (2) has been done using the fourth order Runge-Kuta algorithm with the parameters values $L=3 H, C=1 F, \alpha=0.9, \beta=10.1$ and $\gamma=0.4$ for Fig.(1-a) and $L=3 H, C=1 F, \alpha=0.9, \beta=3$ and $\gamma=0.4$ for Fig.(1-b)

\section{FRACTIONAL-ORDER SIMPLEST MEMRISTOR-BASED CHAOTIC CIRCUIT}

In this paper we present the fractional-order memristorbased circuit and investigate its dynamics by mean of stability theory and numerical schemes.

\section{A. Circuit description and fractional model}

In order to build the fractional-order memristor-based circuit we replace the electrical elements (capacitor, inductor and memristor) in the original circuit by its fractional version (fractional-order capacitor, fractional-order inductor and fractional order memristor) see Fig. 2. Based on Curie's empirical law of 1889 Westerlund et al. proposed in 1994 a fractional-order linear capacitor model and a fractional-order inductor [12], [13]. For a general input voltage $V_{F C}(t)$ the current through the fractional-order capacitor is

$$
I_{F} C(t)=C D^{q_{1}} V_{F C}(t) .
$$

Then

$$
D^{q_{1}} V_{F C}(t)=\frac{1}{C} I_{F L}(t) .
$$

The constant $q_{1}$ is related to the losses of the capacitor. It should be noticed that losses and dissipation are not always the same thing. Dissipation means generation of heat. By losses we imply energy lost from the process under study and not necessarily in the form of heat [12].

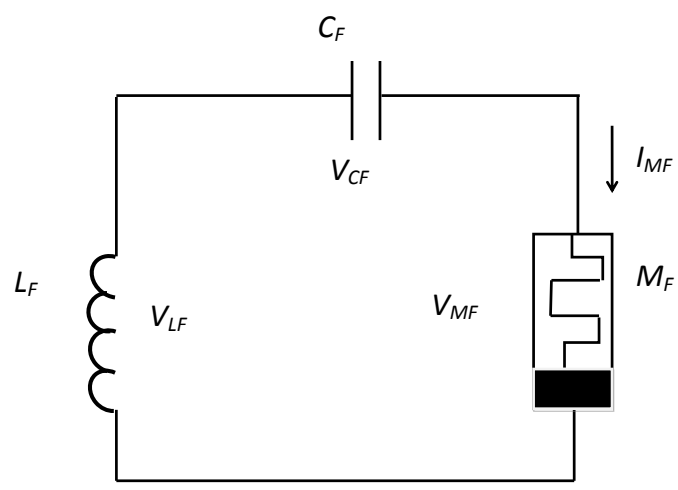

Fig. 2. Fractional-order circuit schematic

For a general current through the fractional-order inductor the voltage is

$$
V_{F L}(t)=L D^{q_{2}} I_{F L}
$$

Then

$$
D^{q_{2}} I_{F L}=\frac{1}{L} V_{F L}(t) .
$$

The constant $q_{2}$ is related to the "proximity effect". When an alternating current flows through an electrical conductor, the current distribution is not uniform. One of the most important electromagnetic phenomenons that dramatically affects the current distribution within any current-carrying conductor, is the electromagnetic proximity effect. A table of various coils and their real orders $q_{2}$ is described in [14]. For a general current through the fractional-order memristor the voltage is [15]

$$
\left\{\begin{aligned}
V_{F M} & =M(z(t)) i_{F M}(t), \\
D^{q_{3}} z(t) & =i_{F M}(t)-\alpha z(t)+i_{F M}^{2} z(t) .
\end{aligned}\right.
$$

Applying Kirchhoff's voltage law we obtain

$$
D^{q_{2}} i_{F L}(t)=-\frac{1}{L}\left(V_{F C}(t)+M(z(t)) i_{F L}(t)\right) .
$$

Using the previous notations of state variable we obtain

$$
\left\{\begin{array}{l}
D^{q_{1}} x=a y \\
D^{q_{2}} y=-b(x+M(z)) y \\
D^{q_{3}} z=-y-\alpha z+y^{2} z
\end{array}\right.
$$

\section{B. Stability analysis}

In this subsection the parameters are set to $a=1$, $b=1 / 3, \alpha=0.9, \gamma=0.4$, with $\beta>0$. we consider the case where all the fractional orders have the same value $q_{1}=$ $\left.q_{2}=q_{3}=q \in\right] 0,2[$ are considered as control parameters. By setting the left-hand side of Eq. (4) to zero, we obtain the only one equilibrium point $E=(0,0,0)$. The stability of E can be investigated using the theorem 2 in [16] and the proposition 2.3 in [17].

Theorem 1: The fractional-order system (4) is asymptotically stable if all the eigenvalues $\lambda$ of the Jacobian matrix $J$ satisfy the condition

$$
|\arg (\lambda)|>\frac{q \pi}{2}
$$




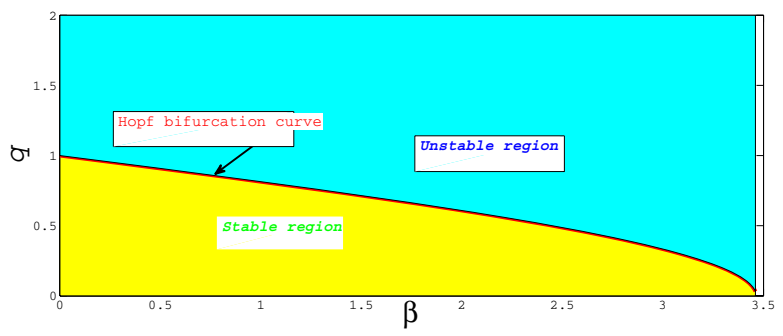

Fig. 3. Stability region of the fractional-order system (4) in the $(\beta-q)$ plane

The Jacobian matrix of system (4) at $E$ is

$$
\left(\begin{array}{ccc}
0 & 1 & 0 \\
-\frac{1}{3} & \frac{\beta}{3} & 0 \\
0 & -1 & -0.9
\end{array}\right)
$$

Its characteristic equation is

$$
\lambda^{3}-\left(\frac{\beta}{3}-0.9\right) \lambda^{2}+\frac{1-\beta}{3} \lambda+0.3=0,
$$

or equivalently

$$
(\lambda+0.9)\left(\lambda^{2}-\frac{\beta}{3} \lambda+\frac{1}{3}\right)=0 .
$$

The eigenvalues are $\lambda_{1}=-0.9<0$ and

- if $\beta \in\left[2 \sqrt{3},+\infty\left[\right.\right.$, then $\lambda_{2,3}=\frac{\beta \pm \sqrt{\beta^{2}-12}}{6}>0$, in this case $E$ is unstable for every value of the commensurate order $q \in] 0,2[$,

- $\quad$ if $\beta \in] 0,2 \sqrt{3}\left[\right.$, then $\lambda_{2,3}=\frac{\beta \pm j \sqrt{12-\beta^{2}}}{6}$, in this case $E$ is asymptotically stable for the values of $q$ and $\beta$ satisfying, $q<\frac{2}{\pi}\left|\tan ^{-1}\left(\frac{\sqrt{12-\beta^{2}}}{\beta}\right)\right|$ and unstable for the values of $q$ and $\beta$ satisfying $q<\frac{2}{\pi}\left|\tan ^{-1}\left(\frac{\sqrt{12-\beta^{2}}}{\beta}\right)\right|$

The stable and unstable regions in the $(\beta-q)$ plane Fig.3, are separated by the curve of equation $q=$ $\frac{2}{\pi}\left|\tan ^{-1}\left(\frac{\sqrt{12-\beta^{2}}}{\beta}\right)\right|$.

\section{Bifurcation analysis}

In [18] we have proposed a fractional-order Hopf bifurcation conditions which state that the system (4) undergoes a Hopf bifurcation through the equilibrium $E$ at the value $\beta^{*}$ of $\beta$ if

i) the Jacobian matrix has two complexconjugate eigenvalues $\lambda_{2,3}$ and one real

$$
\lambda_{1}<0 \text {, }
$$

ii) $\quad m_{2,3}\left(q, \beta^{*}\right)=0$,

iii) $\left.\frac{\partial m_{2,3}}{\partial \beta}\right|_{\beta=\beta^{*}} \neq 0$,

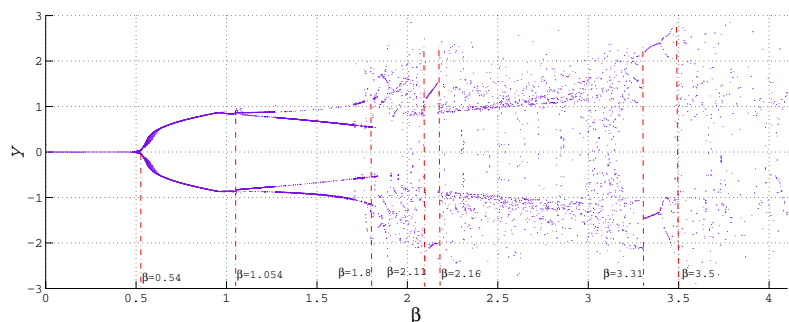

Fig. 4. Bifurcation versus the parameter $\beta$ for $q=0.9$

where

$$
m_{i}(q, \beta)=q \frac{\pi}{2}-\left|\arg \left(\lambda_{i}(\beta)\right)\right|, \quad i=1,2,3 .
$$

If $\beta \in] 0,2 \sqrt{3}[$. Then, the first condition is satisfied. Namely we have $\lambda_{1}=-0.9<0$ and $\lambda_{2,3}=\frac{\beta \pm j \sqrt{12-\beta^{2}}}{6}$.

For $\beta^{*}$ solution of $m_{2,3}\left(q, \beta^{*}\right)=0$ we have

$$
\left.\frac{\partial m_{2,3}}{\partial \beta}\right|_{\beta=\beta^{*}}=\left(\frac{1}{1+\frac{12-\left(\beta^{*}\right)^{2}}{\left(\beta^{*}\right)^{2}}}\right)\left(\frac{-24}{2\left(\beta^{*}\right)^{2} \sqrt{12-\left(\beta^{*}\right)^{2}}}\right) \neq 0 .
$$

Then, all the proposed conditions are satisfied for every solution of $m_{2,3}\left(q, \beta^{*}\right)=0$.

For example if $q=0.9$, then, $\beta^{*}=0.5419$. is a Hopf bifurcation point.

If we consider the fractional order $q$ as a control parameter then we have $\left.\frac{\partial m_{2,3}}{\partial q}\right|_{q=q^{*}}=\frac{\pi}{2} \neq 0$.

Hence, all the solutions $q^{*}$ of $m_{2,3}\left(q^{*}, \beta\right)=0$ with $\beta \in$ ]0, $2 \sqrt{3}[$ are Hopf bifurcation points.

For example if $\beta=3$, we obtain that, $q^{*}=\frac{1}{3}$ is a Hopf bifurcation point. In order to illustrate the above results we present some numerical results.

1) Bifurcation versus the parameter $\beta$ : Fig.4 illustrates the bifurcation diagram of $y$ versus the parameter $\beta$ over the range $0<\beta \leq 4$ where the fractional-order is $q=0.9$. From this figure we can see that when $0<\beta \leq 0.54$ the equilibrium point $E$ is locally asymptotically stable (stationary behavior), when $0.55<\beta<1.83$ the equilibrium point $E$ is unstable and the system exhibits a periodic behavior, which is in agreement with the theoretical results,furthermore the cycle created via Hopf bifurcation, bifurcate in turn when $\beta=1.054$ and two period-one limit cycles appear (coexistence of two limit cycles for $\beta \in] 1.054,1.83$ [ as shown in figure 5 ). When $\beta \in] 1.83,2.11[\cup] 2.16,3.31[\cup] 3.5,3.54[$ the system exhibits a chaotic behavior, alternated with a periodic behavior for $\beta \in] 2.11,2.16[\cup] 3.31,3.5[$.

2) Bifurcation versus the parameter q: Fig.6 illustrates the bifurcation diagram of $y$ versus the fractional-order $q$ over the range $0<q \leq 1.7$. On this figure we can see that when $0<q \leq \frac{1}{3}$ the equilibrium point $E$ is locally asymptotically stable (stationary behavior), when $0.33<q<0.89$ the equilibrium point $E$ losses its stability and the system exhibits a periodic behavior, which is in agreement with the theoretical results, when $0.89<q \leq$ 


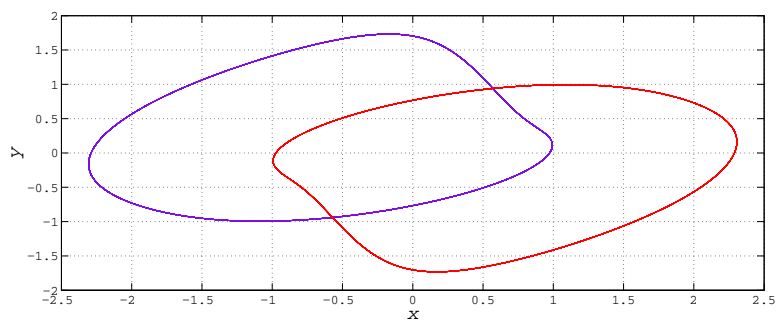

Fig. 5. Coexistence of two period-one limit cycles for $\beta=1.5$ and $q=0.9$

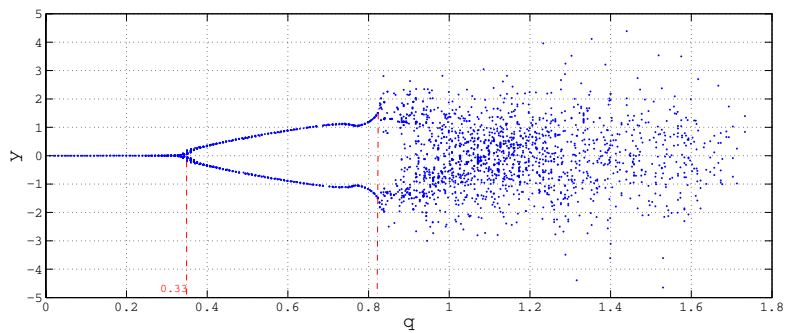

Fig. 6. Bifurcation versus the fractional-order $q$ for $\beta=3$

1.6 the system exhibits a chaotic behavior. Note that here when varying the fractional order the system can display a double scroll chaotic attractor or a four scroll chaotic attractor. Furthermore when $1.4<q<1.7$ the system can display a new chaotic attractor not observed in the integer order case see Fig.7.

\section{The "0-1" test for detecting the chaos}

An efficient binary test for chaos, called " $0-1$ test", has been recently proposed [19] and applied to fractional systems in [20]. The idea underlying the test is to construct a random walk-type process from the data and then to examine how the variance of the random walk scales with time. Specifically, consider a set of discrete data, sampled at times $n=1,2,3, \ldots$, representing a one-dimensional observable data set obtained from the system dynamics. This algorithm consists of the following steps

1) Choose a random value $c \in\left(\frac{\pi}{5}, \frac{4 \pi}{5}\right)$ and define the new coordinates

$$
P_{c}(n)=\sum_{j=1}^{n} \phi(j) \cos (\theta(j))
$$

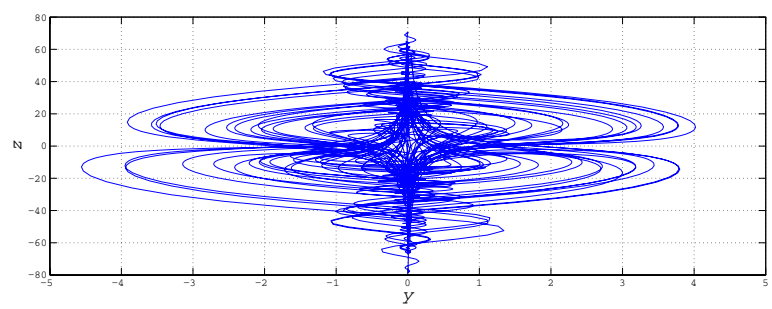

Fig. 7. A new chaotic attractor for $\beta=3, \quad q=1.6$

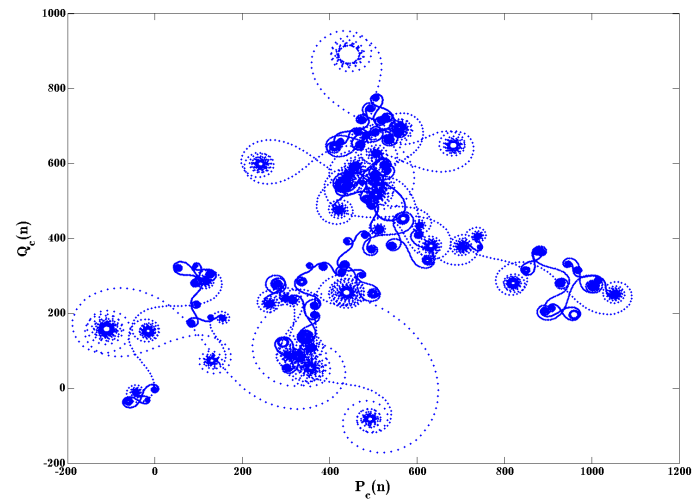

Fig. 8. Unbounded Brownian-like trajectories indicating chaos for $q=1.6$,

and

$$
Q_{c}(n)=\sum_{j=1}^{n} \phi(j) \sin (\theta(j)) .
$$

Where $\theta(j)=j c+\sum_{i=1}^{j} \phi(i), \quad j=1,2,3, \ldots n$.

2) Calculate the mean square displacement

$$
\begin{array}{r}
M_{c}(n)=\lim _{N \rightarrow \infty} \frac{1}{N} \sum_{j=1}^{N}\left(P_{c}(j+n)-P_{c}(j)\right)^{2} \\
+\left(Q_{c}(j+n)-Q_{c}(j)\right)^{2},
\end{array}
$$

where $n \in\left[1, \frac{N}{10}\right]$

$3)$ Define: $K=\operatorname{median}\left(K_{c}\right)$ where

$$
K_{c}=\frac{\operatorname{cov}(\xi, \Delta)}{\sqrt{\operatorname{var}(\xi) \operatorname{var}(\Delta))}} \in[-1,1]
$$

with

$\xi=\left(1,2,3, \ldots n_{\text {cut }}\right), \quad \Delta=\left(M_{c}(1), M_{c}(2), \ldots M_{c}\left(n_{\text {cut }}\right)\right)$

and $n_{\text {cut }}=\operatorname{round}\left(\frac{N}{10}\right)$

4) Interpret outputs: When $K$ is close to 0 , the motion is classified as regular (i.e. periodic or quasi-periodic); when $K$ is close to 1 , the motion is classified as chaotic.

Besides the computation of the rate $K$, the inspection of the dynamics of the $\left(P_{c}(n), Q_{c}(n)\right)$ trajectories provides a simple visual test of whether the system dynamics is chaotic or not. Namely, bounded trajectories in the $\left(P_{c}(n), Q_{c}(n)\right)$ plane imply regular dynamics, whereas Brownian-like (unbounded) trajectories imply chaotic dynamics [19].

In order to analyse the dynamic of the fractional system (4), the " $0-1$ test" has been applied directly to the time series data.

For $q=1.6$, we obtain $K=0.877 \approx 1$. Then the dynamics is chaotic. Moreover, Fig. 8 depicts Brownian-like (unbounded) trajectories in $\left(P_{c}(n), Q_{c}(n)\right)$ plane.

For $q=0.7$, we obtain $K=-0.012 \approx 0$. Then the dynamics is regular. Moreover, Fig. 9 depicts bounded trajectories in the $\left(P_{c}(n), Q_{c}(n)\right)$ plane. 


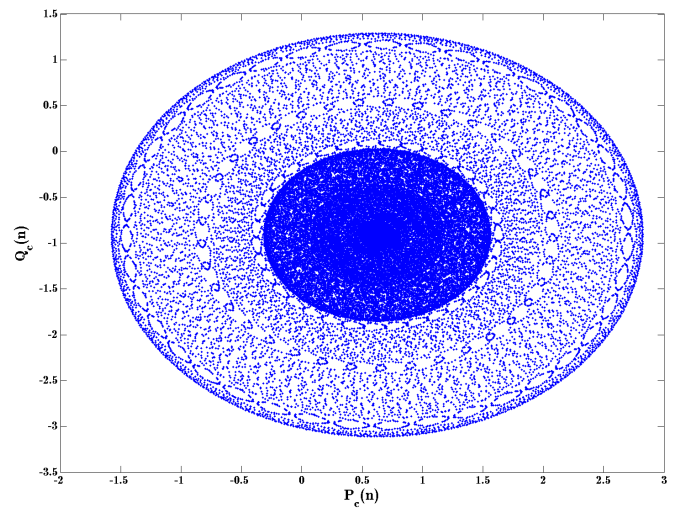

Fig. 9. Bounded trajectories indicating regular dynamic for $q=0.7$,

\section{CONCLUSION}

In this work, we have investigated the dynamical behaviors of the simplest fractional-order electrical circuit that utilizes only three elements in series including a memristor. A second degree polynomial memristance and a second order exponent internal state are used in this circuit to increase complexity of the attractor. A theoretical analysis of the system dynamics has been performed using phase portraits, bifurcation diagrams and the $0-1$ test that has confirmed the presence of chaos in the considered system.

\section{REFERENCES}

[1] D. Cafagna, "Fractional calculus: a mathematical tool from the past for present engineers," in IEEE Ind. Electron. Mag. 1, 2007, pp. $35-40$.

[2] R. Hilfer, Applications of Fractional Calculus in Physics. World Scientific, Singapore, 2000.

[3] H. Sun, A. Abdelwahed and B. Onaral, "Linear approximation for transfer function with a pole of fractional order," in IEEE Trans. Autom. Control 29, 1984, pp. $441-444$.

[4] I. Podlubny, Fractional differential equations. Academic Press, San Diego, 1999.

[5] M. S. Tavazoei and M. Haeri, "A proof for non existence of periodic solutions in time invariant fractional order systems," in Automatica. 45, 2009, pp. 1886 - 1890.

[6] M-S. Abdelouahab and N. Hamri, "The Grunwald-Letnikov Fractional-Order Derivative with Fixed Memory Length," in Mediterranean Journal of Mathematics, doi 10.1007/s00009-0150525-3, 2015, pp. 1-16.

[7] M-S. Abdelouahab, R. Lozi and L.O. Chua, "Memfractance: A Mathematical Paradigm for Circuit Elements with Memory," in Int. J. Bifurc. Chaos, Vol. 24, No. 9, 2014, 29 pages.

[8] L. O. Chua, "Memristor-the missing circuit element," in IEEE Transactions on Circuit Theory. 18, 1971, pp. $507-519$.

[9] D. B. Strukov, G. S. Snider, D. R. Stewart, and R. S. Williams,"The missing memristor found," Nature. 453, 2008,pp. $80-83$.

[10] B. Muthuswamy and L. O. Chua, "Simplest chaotic circuit,"in Int.J. Bifurc. Chaos. 20(5), 2010, pp. $1567-1580$.

[11] L. Teng , H. H. C. Iu , X. Wang and X. Wang, "Chaotic behavior in fractional-order memristor-based simplest chaotic circuit using fourth degree polynomial," in Nonlinear Dyn. 77, 2014, pp. $231-241$.

[12] S. Westerlund and S. Ekstam, "Capacitor theory," in IEEE Transactions on Dielectrics and Electrical Insulation. vol. 1 (5), 1994, pp. $826-839$.
[13] S. Westerlund, "Dead Matter Has Memory!," Kalmar, Sweden: Causal Consulting, 2002.

[14] I. Schafer and K. Kruger, "Modelling of lossy coils using fractional derivatives," in J. Phys. D: Appl.Phys. vol. 41, 2008, pp. $1-8$.

[15] C. Coopmans, I. Petras and Y.Q. Chen, "Analogue fractional order generalized memristive devices," In Proc. of the ASME 2009 International Design Engineering Technical Conferences and Computers and Information in Engineering Conference, IDETC/CIE 2009 August 30 - September 2, 2009, San Diego, California, USA, DETC 2009-86861.

[16] D. Cafagna and G. Grassi, "On the simplest fractional-order memristor-based haotic system," in Nonlinear Dyn. 70, 2012, pp. $1185-1197$.

[17] M-S. Abdelouahab, N.Hamri and J. Wang, "Chaos Control of a Fractional-Order Financial System," in Mathematical Problems in Engineering,2010, doi:10.1155/2010/270646, 18 pages.

[18] M-S. Abdelouahab , N. Hamri and J. Wang, "Hopf bifurcation and chaos in fractional-order modified hybrid optical system," in Nonlinear Dyn. 69, 2012, pp. 275 - 284.

[19] G. A. Gottwald and I. Melbourne, "A new test for chaos in deterministic systems," in Proc. R. Soc. Lond. A 460, 2004, pp. $603-611$.

[20] D. Cafagna and G. Grassi, "Hyperchaos in the fractional-order Rössler system with lowest-order," in Int. J. Bifurc. Chaos. 19, 2009, pp. $339-347$. 